\title{
ENERsip: M2M-based platform to enable energy efficiency within energy-positive neighbourhoods
}

\author{
Gregorio López ${ }^{1}$, Pedro Moura ${ }^{2}$, José Ignacio Moreno ${ }^{1}$, Aníbal de Almeida ${ }^{2}$ \\ ${ }^{1}$ Universidad Carlos III de Madrid, Leganés, Spain, \{gregorio.lopezljoseignacio.moreno\}@uc3m.es \\ ${ }^{2}$ Institute of Systems and Robotics - University of Coimbra, Coimbra, Portugal, \{pmoura|adealmeida\}@isr.uc.pt
}

\begin{abstract}
This paper presents the main features of the EU R\&D project ENERsip. The main objective of this FP7 ICT project is to design, develop, and test an adaptive service-oriented M2Mbased platform that enables optimizing, in near real-time, and saving energy by remotely monitoring, controlling and coordinating power generation and consumption within neighborhoods with energy-positive buildings. The paper describes the system architecture designed to meet such requirements, putting special emphasis on the M2M communication infrastructure. The novel services that will be delivered on top of such M2M communication infrastructure and the potential users who will enjoy them are also presented. Finally, the ENERsip platform validation plan is outlined.
\end{abstract}

Keywords: Addressing; Architecture design; Communication technologies; Services; Validation

\section{INTRODUCTION}

Over the last few decades, worldwide energy demand has increased due to industrial development and global economic growth, resulting in a simultaneous increase in global energy costs and in environmental impacts. Although significant improvements regarding energy efficiency have been achieved in appliance technologies, the end-use electricity consumption has still increased, particularly in buildings. Some of the reasons for such increase are associated with a higher degree of basic comfort and level of service and amenities, as well as with the widespread utilization of new types of loads.

The electronic loads, mostly entertainment and ICT (Information and Communications Technology), consume more than $21 \%$ of the electricity in EU (European Union) houses, a significant percentage of which is standby and other non-active modes (e.g. a router left on $24 \mathrm{hrs}$. per day) [1]. In the EU, the average standby electricity consumption is about $180 \mathrm{kWh}$ per household per year, which is about $7 \%$ of the total annual electricity consumption per household. According to the IEA (International Energy Agency), by $2030,15 \%$ of the total appliance electricity consumption in Europe could be due to standby functions. Therefore, real-time monitoring and control systems are needed to provide the consumers with tools to be aware and to reduce such standby consumptions.

The baseline load consumption of an EU average household is also quite high, near $200 \mathrm{~W}$, mostly due to the cooling appliances and the HVAC (Heating, Ventilating, and Air Conditioning) loads [1]. Washing and drying appliances consume more than $16 \%$ of the electricity with high consumption at peak hours; whereas they may be shifted to other periods. However, if properly controlled, such loads can also be used as a DR (Demand Response) resource. In a scenario of large scale penetration of renewable production, resulting from the EU targets, the DR technologies can be an appropriate mean to compensate the effects of the variability and randomness of the renewable resources availability (e.g. wind and solar intermittence) [2].

Nowadays, the electric grids are evolving towards an increasing presence of electricity generation sources in the buildings (e.g. solar panels), which are used either to generate electricity for own consumption or for selling it to an electric grid operator [3]. According to this scenario, a consumer could also become a producer of electrical energy, i.e. a Prosumer, as coined by Alvin Toffler, and monitoring and control systems will be needed to determine the most profitable option (consumption or grid injection of the produced energy).

M2M communications will play a key role on achieving the above mentioned goals in today's and future Smart Grids scenarios, since they will enable the required bidirectional communication between the high volume of devices to be monitored and controlled and the end users and the information systems where the intelligence resides.

The objective of this paper is to present the ENERsip project, funded by the FP7 (Seventh Framework Programme) ICT and Energy theme, which aims to design, develop, and validate a service-oriented M2M-based energy monitoring and control system for energy grids and decision makers, allowing energy consumption reduction by increasing the consumption awareness of the users and by coordinating their needs with the in-buildings and neighborhoods positive-energy generation facilities. The scope of the ENERsip project can be bounded to the Customer, Distribution, Service Provider, and Operations domains defined in the NIST (National Institute of Standards and Technologies) Smart Grid conceptual model [4].

The remainder of the paper is structured as follows. Section II presents the overall ENERsip architecture as well as each ENERsip domain separately, paying special attention to the M2M communication infrastructure. Thus, section II also presents the most important features of this $\mathrm{M} 2 \mathrm{M}$ communication infrastructure, such as the communication technologies to be deployed, the security they offer, and how address management and end-to-end addressability is handled. Section III describes the ENERsip potential users and the novel services that the platform will offer to them. Finally, section IV draws conclusions and outlines the validation plan of the ENERsip platform.

The research leading to these results has received funding from the European Community's Seventh Framework Programme FP7/2007-2013 under grant agreement $n^{\circ} 247624$. 


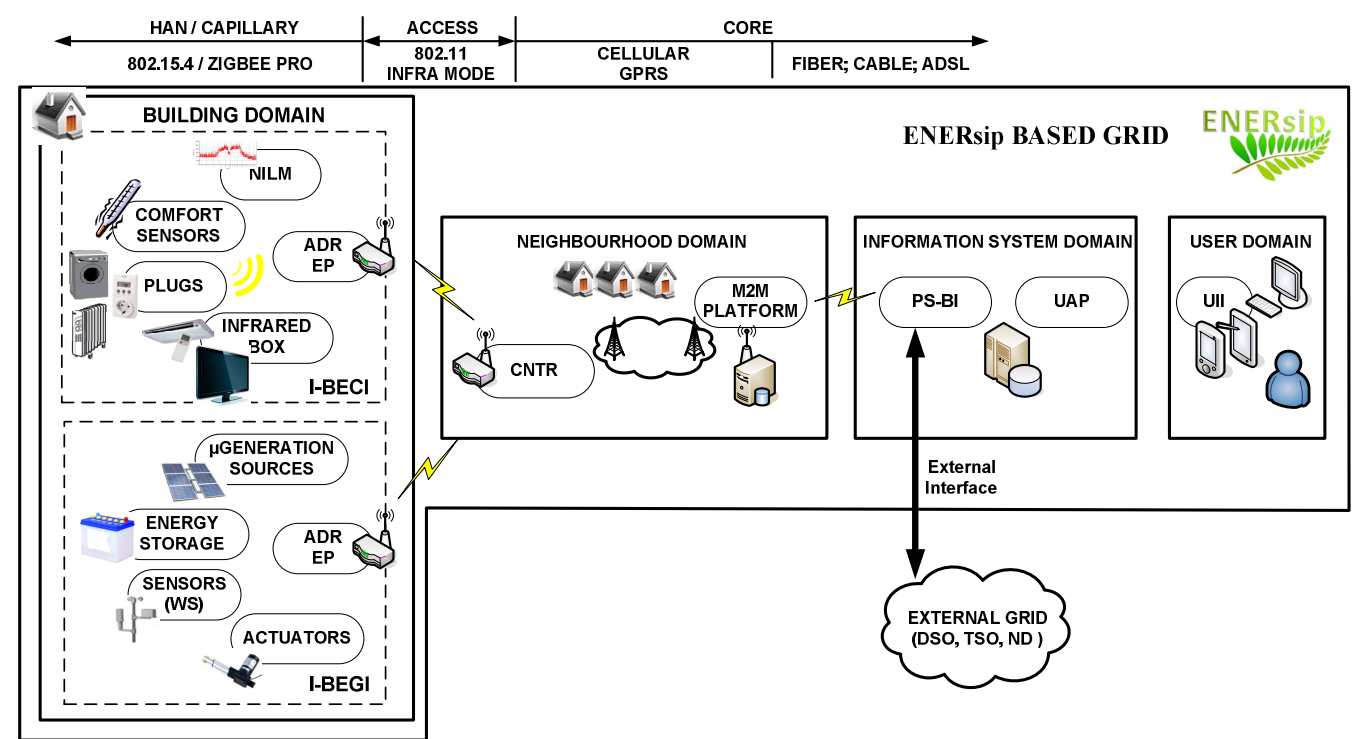

Figure 1 - ENERsip Architecture

\section{THE ENERSIP ARCHITECTURE}

In order to define a fine-grained architecture that helps to break down the development and implementation complexity of the ENERsip platform, the ENERsip system is divided into domains, subsystems and functional blocks, as shown in Fig. 1.

Fig. 1 also illustrates that the ENERsip platform relies on a hybrid and hierarchical M2M communication infrastructure, which is spread mainly across the Building and Neighbourhood Domains. It is hybrid since it is based on different communication technologies depending on the specific requirements of each communication segment. It is hierarchical due to fact that there are specific devices which manage the communications within each communication segment.

\section{A. The ENERsip Domains}

\section{1) Building Domain}

The Building Domain comprises the entire infrastructure associated to smart buildings. The Building Domain is further divided into the I-BECI (In-Building Energy Consumption Infrastructure) and the I-BEGI (In-Building Energy Generation Infrastructure).

The main I-BECI functional blocks are: the Comfort Sensors, the Plugs, the Infrared Box, and the NILM (NonInvasive Load Monitoring) module. The Comfort Sensors measure different environmental variables and transmit them to the Information System (IS) in order to aid energy saving without impacting in-home comfort levels.

The Plugs are associated to "dumb" devices, such as refrigerators or electrical heaters. They act on their power supply by cutting it off or on. They also measure their energy consumption and send it to the IS.

The Infrared Box controls the status of infrared-based "smarter" devices, such as HVAC, TV, or DVD. The proper control of the HVAC systems is especially important for energy saving purposes, since these equipments have large electrical consumption.
The NILM module represents a key element to enable backwards compatibility within the ENERsip platform. Its main goal is to identify (based on electrical signature) and measure the energy consumption of those appliances which are not equipped with a plug with sensor and communication capabilities. The theoretical NILM approach determines when a specific appliance is turned on based just on its electrical signature, by applying non-supervised Digital Signal Processing methods to the overall electrical signal [5]. However, due to the complexity of such methods, in the ENERsip project the user will inform the NILM module about the appliance which has just been turned on in order to help it to learn its electrical signature.

The I-BEGI is composed of $\mu$ Generation Sources, Energy Storage Stations, and a set of Sensors and Actuators. The $\mu$ Generation Sources considered within the ENERsip project are mainly photovoltaic panels and $\mu$ wind mills. The Energy Storage Stations are able to store the exceeding energy for further usage when it cannot be neither injected in the electrical grid nor consumed by the appropriate I-BECI. The I-BEGI actuators are mainly inverters and relays that will properly control the $\mu$ Generation Sources and the Energy Storage Stations respectively. The I-BEGI sensors will be, in general, integrated into Weather Stations (WS). The main objective of these WS is to measure variables related to weather conditions in order to provide the IS with relevant parameters for accurate energy generation forecast.

The I-BECI and the I-BEGI represent the HANs (Home Area Networks), also known as Capillary Networks, within the M2M communication infrastructure. Every single I-BECI or IBEGI will be provided with a gateway, namely the ADR EP (Automated Demand Response End Point). The ADR EPs manage the communication with the in-building energy consumption-generation devices and enable them to get connected to the rest of the ENERsip platform. Thus, the ADR EPs forward the data coming from the in-building energy consumption-generation devices to the IS through the Concentrators (CNTRs) and route the commands coming from the IS to the appropriate device. 


\section{2) Neighbourhood Domain}

The Neighbourhood Domain represents the core M2M infrastructure which allows controlling, monitoring, and managing a high volume of generation and consumption devices spread over a wide area remotely. Therefore, it is responsible for carrying, in a reliable manner, data coming from the Building Domain to the Information System Domain and control and management commands going from the Information System Domain to the Building Domain

The Neighbourhood Domain comprises basically a set of Concentrators (CNTRs) and the M2M Platform. Each CNTR manages a group of ADR EPs (e.g. ID assignment, IP assignment, remove/add ADR EPs). In addition, it forwards the data coming from the ADR EP to the IS through the M2M Platform and routes the commands coming from the IS to the appropriate ADR EP. The communication segment composed by the ADR EPs and the CNTRs represents the Access Network of the M2M communication infrastructure.

The M2M Platform handles the communications with the entire network of CNTRs. On the one side, the M2M Platform is responsible for managing and monitoring the connections with the CNTRs (e.g. ID assignment, IP assignment, remove/add CNTR). On the other side, it forwards all the data coming from the CNTRs to the IS and routes the commands coming from the IS to the appropriate CNTR. The communication segment composed by the CNTRs and the M2M Platform represents the Core Network of the M2M communication infrastructure.

\section{3) Information System Domain}

The Information System Domain is where the intelligence of the electrical grid is residing. The Information System is divided into two different modules: the PS-BI (Power Saving Business Intelligent) and the UAP (User Application Platform). The PS-BI collects (through the M2M communication infrastructure) all the data regarding both energy generation and consumption, processes them, and enables an efficient use of available resources anytime. The UAP enables the provision of a whole set of value-added energy efficiency, comfort monitoring and optimization services based on the user profile and the information provided by the PS-BI.

In addition, the IS may implement a standard interface to enable ENERsip-based grids to interact with bigger electrical infrastructures, such as DSOs (Distribution System Operators), TSOs (Transport System Operators) or NDs (National Dispatchers), and to be managed and coordinated with other neighbourhood grids, thus providing interoperability and scalability.

\section{4) User Domain}

The User Domain represents the set of applications that will be developed to allow the ENERsip users to interact with the platform. Such functionality will be available via User Intuitive Interfaces (UIIs). Such UIIs will be web applications that will allow interacting easily with the ENERsip platform anywhere and anytime seamlessly.

\section{B. Communication Technologies}

Due to the huge number of devices this kind of systems involves, cost and power consumption are two key constraints to be taken into consideration when deciding the most appropriate technologies for them. On the one side, the cost of deploying such monitoring and control infrastructure has to be lower than the cost of building new peaking power plants. On the other side, the energy consumption of such monitoring and control infrastructure has to be lower than the energy it allows saving. However, some other features, such as data rate or range, which depend on the specific requirements of each communication segment, need also to be considered.

\section{1) HANs / Capillary Networks}

The communication technologies used within the ENERsip HANs are different for the I-BECI and for the I-BEGI. For the I-BECI, wireless communication technologies are preferred over wired, mainly because the deployment cost is lower and their flexibility during the maintenance phase is higher. PLC is the only wired technology which this reasoning does not work for. However, PLC is not technically mature enough yet and so it is not cost competitive. The wireless technologies considered for the I-BECI are: Bluetooth (IEEE 802.15.1), Wi-Fi (IEEE 802.11), and IEEE 802.15.4 [6]. Bluetooth is a communication technology designed to replace wires in the communication of multimedia contents between devices. Although it is a quite inexpensive technology, it is plenty of drawbacks, such as it only supports star topology with limited range (very few meters) and it provides too high data rates at the expense of too high power consumption, which make it not appropriate for the ENERsip I-BECI. IEEE 802.11 is a widely deployed and cheap technology. However, IEEE 802.11 is designed to transmit multimedia contents at high data rate. Therefore, its energy consumption is optimized for transmitting and not for idling; whereas the consumption devices transmit few data from time to time and are in idle mode most of the time. As a result, the most appropriate communication technology for the I-BECI is IEEE 802.15.4, since it is defined to minimize power consumption and cost in applications with low data rates and no latency constraints.

There are two main technologies that rely on PHY/MAC IEEE 802.15.4 standard: 6LoWPAN (IPv6 over Low power Wireless Personal Area Networks) and Zigbee. 6LoWPAN is an open standard that is being defined by the IETF [7]. Its main advantage is that it allows integrating IEEE 802.15.4 networks into the Internet seamlessly. Its main drawback is that it is in the early development phase yet. Zigbee is an open industrial standard developed by the Zigbee Alliance. It has been around in the market for longer time than 6LoWPAN, so it is much more mature. As a matter of fact, the most important hardware and appliance manufacturers, such as Freescale, Texas Instruments or General Electric, currently commercialize Zigbee-capable chips and equipment. In addition, the Zigbee Alliance has already launched the Zigbee Smart Energy Profile, which is specially designed to meet energy efficiency scenarios' requirements and it also incorporates the IP into Zigbee (actually, it incorporates 6LoWPAN into the Zigbee stack). Therefore, the Zigbee PRO feature set [8] is selected for the I-BECI. 
Since developing specific hardware for the I-BEGI is out of the scope of the project, the communication technologies to be used here depend on the available commercial equipment. Thus, the ENERsip generation devices will communicate with their associated ADR EP using IEEE 802.15.4/Zigbee, RS232, RS485, PLC or Ethernet (IEEE 802.3).

\section{2) Access Network}

For the communication between the ADR EPs and the CNTRs, two technologies are considered: IEEE 802.15.4/Zigbee and IEEE 802.11. In this case, IEEE 802.11 is chosen over Zigbee since it clearly fits the requirements of this communication segment better (i.e. IEEE 802.11 provides higher bandwidth and coverage). Since it is a quite static and star topology network, the most appropriate IEEE 802.11 working mode is the infrastructure mode, the CNTRs working as APs (Access Points) and the ADR EPs being the clients.

\section{3) Core Network}

For the communication between the CNTRs and the M2M Platform, the following communication technologies are taken into account. ADSL is considered not to be appropriate for the ENERsip platform due to neighbourhood's network infrastructure installation requirements. The LTE, HSPA + and WiMAX technologies are not considered appropriate mainly because they are not widely deployed yet and so they are not mature enough. HSxPA (WCDMA) presents some advantages, e.g. it offers increased peak data rates and reduced latency. However, its main problem is that it requires some enhancements inside of the infrastructure that are not yet fully spread in Europe. Therefore, GPRS/EDGE is considered the most appropriate technology for this communication segment because it is widely deployed and mature. In addition, GPRS/EDGE is considered to be the available technology that fits the requirements of this kind of systems (in terms of data rate) better.

The communication between the M2M Platform and the IS will be based on broadband wired technologies (e.g. fiber, cable).

\section{Security}

Security and privacy are two key challenging issues for this kind of systems [9]. Although privacy is completely out of the scope of the ENERsip project, it does address security. Next, the main security features of the selected communication technologies are outlined.

\section{1) HANs / Capillary Networks}

Taking into account the relevance of the information managed by the ENERsip platform, the Zigbee PRO High Security Mode [8] is recommended for HANs. Moreover, following the same reasoning, it is strongly recommended to use Master Keys factory installed on the devices, since it reduces dramatically the vulnerability of the wireless network.

\section{2) Access Network}

For the Access Network, IEEE 802.11 using WPA2 is recommended. MAC filtering or AP hiding can be also applied to increase security. VPNs (Virtual Private Networks), configured using IPsec, may be used to provide additional data protection at network layer and above. However, the overhead introduced by this security mechanism has to be carefully taken into consideration.

\section{3) Core Network}

For the GPRS communications, additional security mechanisms controlled by the ENERsip platform may be implemented (e.g. Secure Socket Layer - SSL - over TCP/IP). However, this decision has to be considered carefully again, since encryption means redundancy and so more bits to be transmitted, which in turn means more money to be paid to the Network Operator/Service Provider. Therefore, a trade-off between security and cost has to be reached.

\section{Addressing}

Due to the facts that M2M systems involve a huge amount of devices, that the deployment of this kind of systems is growing really fast, and that predictions point they will grow even faster, efficient numbering and addressing represents definitely a major challenge in M2M communications. The main two trends to tackle this problem are: using E.164 numbers, as short-term solution, and using IPv6 addresses, as long-term solution [10].

The ENERsip approach is closer to the latter, since it is based on IPv4 addresses until the ADR EPs, which are responsible for routing incoming packets to the appropriate consumption-generation end-device by mapping somehow the IPv4 address associated to their IEEE 802.11 interface onto the address space of their HAN. In order to enable such procedure, the ENERsip platform defines its own addressing solution at the application level, which allows identifying univocally and addressing every single ENERsip communication device by taking advantage of the hierarchy of the ENERsip M2M communication infrastructure.

In the ENERsip platform, each device is assigned a 16-bits ID. The scope of this ID is bounded to the communication element which is right above in the hierarchical communication infrastructure. Thus, each M2M Platform has a pool of $2^{16}$ IDs, so it can address univocally up to $2^{16}$ CNTRs; every single CNTR, in turn, can manage up to $2^{16}$ ADR EPs; and, finally, every single ADR EP can manage up to $2^{16}$ consumptiongeneration end-devices. Therefore, each consumptiongeneration end-device is globally univocally identified by the 8-bytes ID which results from concatenating its own 2-bytes ID with its associated ADR EP, CNTR and M2M Platform IDs.

The ID distribution and management in the ENERsip platform is performed as follows. If, in order to increase the geographical area or the number of devices the platform can cover or manage respectively, there is more than one $\mathrm{M} 2 \mathrm{M}$ Platform, each M2M Platform is assigned a 2-byte ID which is stored in the IS together with its associated IMSI (International Mobile Subscriber Identity). Each M2M Platform is equipped with a GPRS SIM (Subscriber Identity Module) card which contains the IMSI and a 128-bits authentication key. These two parameters represent the golden key that allows authenticating and univocally identifying each M2M Platform in the very beginning, i.e., at least, the first time it is switched on.

The CNTRs are provisioned in the appropriate M2M Platform in advance, i.e. the system administrator provides the 
M2M platform with a table that contains the IMSIs of all the CNTRs which are planned to be deployed and their associated 2-bytes IDs. Note that this table is also stored in the IS. When a new CNTR is switched on, it authenticates itself in the appropriate M2M Platform using its IMSI and the 128-bits key. If this procedure is successful (i.e. it is not a fake CNTR), the M2M Platform assigns the CNTR its 2-bytes ID and an IP address. The 2-bytes ID is fixed and it will never change, unless the CNTR is not valid anymore. However, the IP address is assigned dynamically, so it may change from time to time or after rebooting the CNTR.

The ADR EPs are also pre-provisioned in the appropriate CNTRs. The ADR EPs are equipped with multiple communication interfaces. However, they use their IEEE 802.11 MAC address together with a key (which may be preprogrammed on it) to authenticate themselves in the appropriate CNTR, because it is mandatory for the ADR EPs to be equipped with an IEEE 802.11 interface, since this is the only technology used within the Access Network. If this procedure is successful, the CNTR assigns the ADR EP its fixed 2-bytes ID and an IP address which may change dynamically. Note that, since every single ADR EP is associated to a given customer, in this case the IS stores the Customer ID together with all the communication nodes' IDs which lead to its ADR EP (i.e. M2M Platform's ID, CNTR's ID, and ADR EP's ID).

The procedure for registering new consumption-generation end-devices differs depending on the communication technology in use. In any case, the devices have to be registered one by one and they have to authenticate themselves in their associated ADR EP. If the device is Zigbee-capable, for instance, after being switched on by the customer, it authenticates itself in the appropriate ADR EP using its IEEE 802.15.4 64-bits long MAC address and its associated Master Key (see section II.C.1). Then, the ADR EP assigns the device a 16-bits short MAC address (2-bytes ID) and sends the pair [64-bits MAC address, 2-bytes ID] to the IS. The IS first checks if there is already a device associated to this ADR EP which is univocally identified by this 64-bits MAC address. If so, it only updates its associated 2-bytes ID. Otherwise, it creates a new entry for this ADR EP storing the 64-bits MAC address, the 2-bytes ID, as well as other information which is asked the user to fill, such as the type of device (e.g. washing machine, TV, air conditioning), the brand, the model, the location of the device within the house, and a human-friendly name.

The routing of data in the ENERsip platform is performed as follows. When the data flow from the Building Domain to the Information System Domain/User Domain (e.g. sensor reporting a periodic measurement), the procedure is straightforward since every single device in the communication chain has only one option as next hop.

When the data flow from the User Domain/Information System Domain to the Building Domain, the procedure is a bit more complex. For instance, if a given user wants to send a request to a specific appliance, first, the IS will traverse the tree associated to this Customer ID, fetch the appropriate sequence of 2-bytes IDs which represent this appliance, and send the request to the appropriate $\mathrm{M} 2 \mathrm{M}$ Platform, as it is the next hop. The M2M platform will check its table that maps CNTR 2bytes IDs with IP addresses, it will fill the destination IP address properly and it will route the packet to the appropriate CNTR. The CNTR will perform exactly the same procedure to route the packet to the appropriate ADR EP. Finally, the ADR EP will check its table that maps end-device 2-bytes IDs with HAN-valid addresses and it will route the packet to the appropriate appliance.

\section{THE ENERSIP SERVICES}

The ENERsip platform is primarily targeted at owners of residential and commercial buildings, which can be divided into RC (Residential Consumers), RP (Residential Prosumers), and CP (Commercial Prosumers). However, the ENERsip platform will also be used by other stakeholders, such as the LEPs (Local Energy Producers), the ACPs (Aggregators of Consumption and Production), and the DSOs (Distribution and Transmission System Operators). The users will benefit with different services, such as: EMVR (Energy Monitoring, Visualization, and Reporting); RACA (Remote Access and Control of Appliances); LM (Load Management); MEM (Microgrid Energy Management); and DSO (Distribution System Operation). Table I shows the mapping of service types onto user segments.

TABLE I. ENERsip USER SEgMENTS AND SERVICE TyPes MAtCHING

\begin{tabular}{|l|c|c|c|c|c|c|}
\hline & RC & RP & CP & LEP & ACP & DSO \\
\hline EMVR & $\mathrm{X}$ & $\mathrm{X}$ & $\mathrm{X}$ & $\mathrm{X}$ & $\mathrm{X}$ & $\mathrm{X}$ \\
\hline RACA & $\mathrm{X}$ & $\mathrm{X}$ & $\mathrm{X}$ & $\mathrm{X}$ & & \\
\hline LM & $\mathrm{X}$ & $\mathrm{X}$ & $\mathrm{X}$ & & & $\mathrm{X}$ \\
\hline MEM & & $\mathrm{X}$ & $\mathrm{X}$ & $\mathrm{X}$ & $\mathrm{X}$ & \\
\hline DSO & & & & & & $\mathrm{X}$ \\
\hline
\end{tabular}

\section{A. Energy monitoring, visualization, and reporting}

The residential and commercial users will be provided with near real-time information about their energy consumption and / or generation and the associated economic and environmental impact. The ACPs and the DSOs can also receive part of such information, depending of the contract with the prosumer.

In relation to energy generation, beyond the information about the energy generation and prices, the system will also provide the production forecasting, which will be very important for the prosumers to determine the most profitable option (consumption or grid injection of the produced energy). Regarding the energy consumption, the ENERsip platform will also provide detailed information about the consumption of each individual appliance enabling the comparison between the actual energy consumption with the expected one. As a result, any deviations and opportunities to reduce energy consumption (e.g. equipment schedules, appliance upgrades or replacements) will be reported to the users.

\section{B. Remote access and control of appliances}

The ENERsip system will enable the remote control of the in-home or in-building appliances by the users through a webbased interface (UII). Using such interface, the user will have the possibility to create an initial configuration of the network, 
remotely switch on/off devices, or to adjust their settings. In addition, the user will be allowed to configure rules that will apply pre-defined actions (e.g. switch off, adjust set point, delay start) to specific devices according to dynamic changes of the overall context. Such control will give the user the possibility of adapting the devices operation to the user needs, to changes of weather conditions or to real-time prices. With the remote monitoring, the user can identify appliances which are unnecessarily turned on and turn them off with the remote control, avoiding unnecessary consumption. Additionally, the remote control capability will facilitate the participation in DR programs.

\section{Load management}

In the future electricity market it will be important for each end user to have the ability to respond quickly to dynamic changes in the market, such as real-time prices. The load management functionality offered by the ENERsip platform will also enable the participation of the users in DR programs. The user will be able to specify the devices to be included in the automated load management program and manually override it. The load management capability will bring economic advantages to the users and additional control and optimization capabilities to the DSOs.

\section{Microgrid energy management}

The microgrid energy management service will work at the local communities and neighbourhoods level, where multiple consumption, generation and storage units might be running. Since the LEGs are mostly renewable sources with variable and intermittent production, the optimization process at the microgrid level in an economic and reliable perspective is not an easy operation. Therefore, the monitoring capability of the generation and consumption will increase the real-time knowledge of the conditions and contribute to improve the balance between generation and consumption in the microgrid.

\section{E. Distribution system operation}

The ENERsip platform can offer near real-time information about distributed generation and consumption of electricity in a given locality, highlight the deviations from the expected behaviour and provide accurate short-term forecasts of the LEG. Such information will be very important for the DSOs to the planning and dispatching of the generation resources. However, the most important impact of this service is to obtain the required conditions to operate DR programs, which force consumption reductions at near real time in critical situations.

\section{CONCLUSION}

This paper presents current status and main research lines of the FP7 ICT project ENERsip, whose main objective is to design, develop, and test a smart, open, and service-oriented platform to reduce energy consumption and to match energy generation and consumption within neighbourhoods with energy-positive buildings.

In order to achieve this goal, the ENERsip platform tightly relies on a M2M communication infrastructure. Throughout this paper, the architecture and main features (i.e. communication technologies, security, and addressing) of such M2M communication infrastructure are described.

As a result, the paper illustrates how important M2M communications are to enable energy efficiency in Smart Grids. Some of the benefits, in terms of services, that M2M communications can bring to this area as well as the potential users who will enjoy them are also presented.

The ENERsip platform will be validated in two phases. The first validation phase will take place in 2011 in the ISR-UC facilities (Coimbra, Portugal). This validation phase will check the functionality and the integration of the developed pieces of hardware and software. The second validation phase will take place in 2012 in the IEC facilities (Haifa, Israel) and it will test the ENERsip system as a whole. In order to evaluate some figures-of-merit such as performance, scalability, or the impact of the communication infrastructure on the achieved energy efficiency, this test bed will consist of a pilot scheme, composed of real hardware, and regular PCs that will emulate in-building energy consumption and generation devices, ADR EPs, CNTRs and the behavior of the communication links among them all.

\section{ACKNOWLEDGMENT}

The authors would like to thank the whole ENERsip consortium: Amplía Soluciones S.L, AC\&A (Aplicaciones de Control y Automatización), Honeywell SPOL, ISA (Intelligent Sensing Anywhere), IEC (Israel Electric Corporation), ISR-UC (Institute of Systems and Robotics - University of Coimbra), MIL (Motorola Israel Ltd.), TECNALIA, UC3M (Universidad Carlos III De Madrid), and VITO (Vlaamse Instelling voor Technologisch Onderzoek).

\section{REFERENCES}

[1] REMODECE, 2008. "REMODECE (Residential Monitoring to Decrease Energy Use and Carbon Emissions in Europe) Final Publishable Report", ISR-University of Coimbra. November 2008. Online: www.isr.uc.pt/ remodece.

[2] Moura, Pedro and de Almeida, Aníbal, "The Role of Demand-Side Management in the Grid Integration of Wind Power", Applied Energy, Elsevier, Vol. 87, Issue 8, pag. 2581 - 2588, August 2010.

[3] de Almeida, Aníbal and Moura, Pedro, "Distributed Generation and Demand Side Management", in the Handbook of Conservation and Renewable Energy, CRC Press, John Kreider and Yogi Goswami (ed.), ISBN: 9780849317309, CRC Press, Boca Raton, Florida, USA, 2007.

[4] IEEE Smart Grid Official Site: http://smartgrid.ieee.org/nist-smartgridframework

[5] S. D. Warren, L. D. Brandeis, "Residential Energy Monitoring and Computerized Surveillance via Utility Power Flows", IEEE Technology and Society Magazine, June 1989.

[6] "Wireless Medium Access Control and Physical Layer Specifications for Low-Rate Wireless Personal Area Networks," IEEE Std 802.15.4-2006.

[7] G. Montenegro, K. Kushalnagar, J. Hui, D. Culler, "Transmissions of IPv6 packets over IEEE 802.15.4 Networks.” RFC 4944, September 2007. Online: http://www.rfc-editor.org/rfc/rfc4944.txt

[8] Zigbee Alliance. "Zigbee 2007/PRO specification”, October 2007.

[9] P. McDaniel, S. McLaughlin, "Security and Privacy Challenges in the Smart Grid", IEEE Security and Privacy, vol. 7, no.3, pp.75-77, May/June 2009.

[10] Draft ECC Report 153, "Numbering and Addressing in M2M Communications", Vilnius, June 2010. 